Classification

Physics Abstracts

$71.25 \mathrm{P}-71.45 \mathrm{G}-75.50-79.60$

\title{
Effect of Coulomb correlations on energy bands in ferromagnetic transition metals : $\mathrm{Ni}$, Co and Fe
}

\author{
G. Tréglia (*), F. Ducastelle (*) and D. Spanjaard (**) \\ (*) O.N.E.R.A., 29, avenue de la Division Leclerc, 92320 Châtillon sous Bagneux, France \\ ${ }^{* *}$ Laboratoire de Physique des Solides, Bât. 510, Université Paris-Sud, Centre d'Orsay, 91405 Orsay, France
}

(Reçu le 15 juillet 1981, accepté le 9 octobre 1981)

\begin{abstract}
Résumé. - Nous présentons ici la généralisation au cas ferromagnétique de la méthode de perturbation (au second ordre en $U / W ; U$ : intégrale de Coulomb, $W$ : largeur de la bande d) développée précédemment pour prendre en compte les effets de corrélations dans les métaux paramagnétiques. Nous calculons ainsi les modifications induites par ces effets sur les structures de bande des ferromagnétiques : $\mathrm{Ni}$, Co et $\mathrm{Fe}$. L'accord avec les structures de bande expérimentales (déduites de la photoémission angulaire) est satisfaisant pour les valeurs suivantes de $U: U=2 \mathrm{eV}$ pour $\mathrm{Ni}, U=1,5 \mathrm{eV}$ pour $\mathrm{Co}$ et $U=1 \mathrm{eV}$ pour $\mathrm{Fe}$.
\end{abstract}

\begin{abstract}
We present here the extension to the ferromagnetic case of the perturbation method (limited to second order in $U / W ; U$ : Coulomb integral, $W: \mathrm{d}$ bandwidth) previously developed for taking into account correlation effects in paramagnets. Within this model, we can calculate the modifications of the band structures of ferromagnetic $\mathrm{Ni}, \mathrm{Co}$ and $\mathrm{Fe}$, induced by these effects. A good agreement with « experimental » band structures (deduced from angle resolved photoemission data) is obtained for the following values of $U: U=2 \mathrm{eV}$ for $\mathrm{Ni}, U=1.5 \mathrm{eV}$ for Co and $U=1 \mathrm{eV}$ for Fe.
\end{abstract}

1. Introduction. - Recently there has been several angle resolved photoemission experiments providing direct observation of energy band dispersions in $\mathrm{Cu}$ [1-3] and $\mathrm{Ni}$ [4-7] (for a more general review, see also [8]). Whereas agreement with standard one electron band calculations is good for $\mathrm{Cu}$, the case of $\mathrm{Ni}$ sets some problems : presence of a satellite [9], narrowing of the $d$ bandwidth, reduction of the exchange splitting... These discrepancies have stimulated a large number of theoretical papers [10-19] which all agree in explaining these features by invoking correlation effects within the unfilled $d$ band. Such an interest limited to Ni seems to imply that other transition metals (in particular the other ferromagnets Co and $\mathrm{Fe}$ ) do not exhibit similar behaviours. However, it would be surprising that correlation effects could play such an important rôle in $\mathrm{Ni}$, and no rôle in $\mathrm{Co}$ or $\mathrm{Fe}$ in spite of an increasing number of $\mathrm{d}$ holes (even if this is compensated by a lower value of the Coulomb integral : $U$ ).

In fact, for many reasons, Ni has been much more studied than other transition metals, from an experimental or theoretical point of view. Several independent, but consistent band calculations have been published [20-22], and there are now also several independent angle resolved photoemission data [4-7, 9] which show unambiguous discrepancies with the calculated one electron spectra. Thus, $\mathrm{Ni}$ appears as an archetypal case. Less is known about $\mathrm{Fe}$ and $\mathrm{Co}$; in particular, in the latter case, contradictory results [22-24] have been given concerning the theoretical energy bands or exchange splittings. However recent experiments by Eastman et al. [25] on these metals seem to indicate that problems similar to those found in $\mathrm{Ni}$ are also encountered, at least in the case of $\mathrm{Co}$.

For all these reasons, we present here the extension to the ferromagnetic case of the perturbation method (limited to second order in $U / W-W$ : d bandwidth-) previously developed for taking into account correlation effects in paramagnets [17] (preliminary outlines have already been given in [19, 27]). All other previous theoretical works dealt with $\mathrm{Ni}$. Most of them [10-11] considered a nearly filled $\mathrm{d}$ band and used the $t$-matrix approximation, which may be criticized even in the case of $\mathrm{Ni}$ [18]. Recently Liebsch [12] has presented an improved approximation, but its final formulation still relies on the assumption of a low density system (furthermore, it is not completely clear how the spin dependence is treated). Obviously, this kind of approximation cannot apply 
to $\mathrm{Co}$ and $\mathrm{Fe}$. Another numerical approach has been developed by Davis and Feldkamp [13-14]. It is well suited to a $\mathrm{s}$ band but its extension to degenerate $\mathrm{d}$ bands requires further approximations which have not yet been fully discussed. Finally, in a very recent paper [16], Kleinman and Mednick have performed independently a similar perturbation calculation in the particular case of $\mathrm{Ni}$, which basically justifies our approximations. However, their choice of $U$ differs from ours; this will be discussed later on.

In the present paper, we develop a systematic study of the influence of correlations on the electronic structure of $\mathrm{Ni}, \mathrm{Co}$ and $\mathrm{Fe}$, using the perturbation method alluded to before. This method will be shown to yield results at least as good as the others in the case of $\mathrm{Ni}$; furthermore, it can be applied to any filling of the $d$ band $(\mathrm{Co}, \mathrm{Fe})$. In section 2 we describe our model; then we give the general qualitative results $(\S 3)$ and finally $(\S 4)$ we apply these results to the cases of $\mathrm{Ni}, \mathrm{Co}$ and $\mathrm{Fe}$, and present improved theoretical band structures.

2. Description of the model. - As in previous works [17-19], we use here the Hubbard hamiltonian in which the degeneracy is introduced in the simplest way, i.e. we keep a single average Coulomb interaction $U$ for electrons in different atomic d states $|n, v, \sigma\rangle$ on the same atom $n, v$ and $\sigma$ being orbital and spin indices respectively:

$$
\begin{aligned}
H=H_{0}+\frac{U}{2} \sum_{n, v \sigma, v^{\prime} \sigma^{\prime}}(1- & \left.\delta_{v v^{\prime}} \delta_{\sigma \sigma^{\prime}}\right) \times \\
& \times c_{n, v \sigma}^{+} c_{n, v \sigma} c_{n, v^{\prime} \sigma^{\prime}}^{+} c_{n, v^{\prime} \sigma^{\prime}}
\end{aligned}
$$

where $c^{+}$and $c$ are creation and annihilation operators respectively, and $H_{0}$ is the band hamiltonian in a tight binding model. A conventional HartreeFock scheme leads to an effective one electron hamil- tonian; the remaining interaction term is treated within the second order perturbation theory mentioned above. More precisely, the spectral density $n_{k \sigma}(E)$ is directly related to the imaginary part of the retarded Green function $G_{k \sigma}(E)$ through :

$$
n_{k \sigma}(E)=-\frac{\operatorname{Im}}{\pi} G_{k \sigma}(E)
$$

with :

$$
G_{k \sigma}(E)=\left(E-E_{k \sigma}-\Sigma_{k \sigma}(E)\right)^{-1}
$$

and we calculate the one particle self energy $\Sigma_{k \sigma}(\dot{E})$ up to second order in $U / W . E_{k \sigma}$ is the Hartree-Fock single particle energy which, in the case of ferromagnets, is spin dependent (Hartree-Fock-Stoner model), so that we can define the exchange splitting $\Delta\left(E_{k \downarrow}\right)$ :

$$
\Delta\left(E_{k \downarrow}\right)=E_{k \downarrow}-E_{k \uparrow} .
$$

In the following, the unperturbed one electron band structure will be taken from self-consistent spin polarized band calculations. It has been shown [28] that the latter are quite consistent with a Stoner model in which the exchange splitting is slightly energy dependent (it increases from the bottom to the top of the band : this behaviour is well understood [28]). As indicated in equation (4), this energy dependence is referred to the minority spin band, which will be the case of each energy dependent quantity in the following.

We have already shown [17] that a so-called local approximation is quite reasonable when calculating the self energy which is therefore $k$-independent. $\Sigma_{\sigma}\left(E, U, N_{\mathrm{e}}\right)$ is then obtained from a straightforward extension of formula (14) in reference [17] :

$$
\Sigma_{\sigma}\left(E, U, N_{\mathrm{e}}\right)=U^{2} \Sigma_{\sigma}\left(E, N_{\mathrm{e}}\right)
$$

with :

$$
\begin{aligned}
& \Sigma_{\sigma}\left(E, N_{\mathrm{e}}\right)=4 \int_{m_{\sigma}}^{E_{\mathrm{F}}} \int_{m_{\sigma}}^{E_{\mathrm{F}}} \mathrm{d} E_{3} \mathrm{~d} E_{4} \int_{E_{\mathrm{F}}}^{M_{\sigma}} \mathrm{d} E_{2} f_{\sigma \sigma}\left(E, E_{2}, E_{3}, E_{4}\right)+4 \int_{E_{\mathrm{F}}}^{M_{\sigma}} \int_{E_{\mathrm{F}}}^{M_{\sigma}} \mathrm{d} E_{3} \mathrm{~d} E_{4} \int_{m_{\sigma}}^{E_{\mathrm{F}}} \mathrm{d} E_{2} f_{\sigma \sigma}\left(E, E_{2}, E_{3}, E_{4}\right)+ \\
& \quad+5 \int_{m_{\sigma}}^{E_{\mathrm{F}}} \mathrm{d} E_{4} \int_{m_{-\sigma}}^{E_{\mathrm{F}}} \mathrm{d} E_{3} \int_{E_{\mathrm{F}}}^{M_{-\sigma}} \mathrm{d} E_{2} f_{-\sigma \sigma}\left(E, E_{2}, E_{3}, E_{4}\right)+5 \int_{E_{\mathrm{F}}}^{M_{\sigma}} \mathrm{d} E_{4} \int_{E_{\mathrm{F}}}^{M_{-\sigma}} \mathrm{d} E_{3} \int_{m_{-\sigma}}^{E_{\mathrm{F}}} \mathrm{d} E_{2} f_{-\sigma \sigma}\left(E, E_{2}, E_{3}, E_{4}\right) .
\end{aligned}
$$

and :

$$
f_{\sigma \sigma^{\prime}}\left(E, E_{2}, E_{3}, E_{4}\right)=\frac{n_{\sigma}\left(E_{2}\right) n_{\sigma}\left(E_{3}\right) n_{\sigma^{\prime}}\left(E_{4}\right)}{E+E_{2}-E_{3}-E_{4}}
$$

$E_{\mathrm{f}}$ is the Fermi level ; $m_{\sigma}, M_{\sigma}$ and $n_{\sigma}(E)$ are respectively the bottom, the top and the density of states of the $\sigma$ spin band and $N_{\mathrm{e}}$ is the $\mathrm{d}$ band filling. The numerical factors 4 and 5 correspond to the respective numbers of available scattering channels for $\sigma$ spin electrons in $\sigma$ and $-\sigma$ spin bands. Previous calculations [17] have shown that this self energy is rather insensitive to the details of the density of states $n_{\sigma}(E)$ and in the following we shall use rectangular densities of states with widths fitted to the second moment of the exact curves. Furthermore, in principle, $\Sigma_{\sigma}(E)$ should be symmetry dependent and one should define (for a cubic system) $: \Sigma_{\mathrm{t}_{2 \mathrm{~g}}} \neq \Sigma_{\mathrm{e}_{\mathrm{g}}}$; we shall argue that in practice these differences lead to very small effects. 
One can see from formula (5) that the order of magnitude of $\Sigma\left(E, U, N_{\mathrm{e}}\right)$ is given by the quantity : $\left(N_{\mathrm{e}} / 10\right)$ $\left(1-N_{\mathrm{e}} / 10\right) U^{2} / W$, which implies that the range of values of $U$ for which our perturbation method is valid is larger for nearly filled (empty) bands than for half filled ones.

Finally, our method only requires a few number of parameters : the $\mathrm{d}$ bandwidths $W_{\sigma}$, the $\mathrm{d}$ band filling $N_{\mathrm{e}}$ and the spin magnetic moment $\mu$, which are taken from self-consistent band calculations. Then we have just to fit the Coulomb parameter $U$ by comparing our perturbed band structures to the experimental ones. Actually such a fit will be shown to account also for several other properties, but we shall have to check that $U$ takes reasonable values, i.e. that $U / W$ is at most about unity [18].

3. General trends. - The variations of the self energies $\Sigma_{\sigma}\left(E, N_{\mathrm{e}}\right)$ as calculated from formula (5) are shown in figure 1 for different fillings of the $d$ band. Some general qualitative arguments can be already deduced from the overall behaviour of $\Sigma_{\sigma}$, which are actually necessary consequences of the general Landau theory for Fermi liquids; in other words, an exact theory should yield similar qualitative effects. When $U \rightarrow 0$, the real and imaginary parts of $\Sigma_{\sigma}\left(E, U, N_{\mathrm{e}}\right)$ are exactly the shift and the broadening of the initial Bloch states $E_{k \sigma}^{0}$. It is then easy to see in figure 1 that this broadening increases when going deeper in the band. On an other hand, the shifts are positive (at least for $\mathrm{Ni}, \mathrm{Co}$ and the majority spin band of $\mathrm{Fe}$ ) and they always lead to a narrowing of the upper states $(\mathrm{d} \Sigma / \mathrm{d} E<0$ near the top of the band).

For moderate values of $U / W$, this analysis breaks down since the spectral density $n_{k \sigma}(E)$ (see formula (2)) has no longer a lorentzian shape. This implies that we are using perturbation theory beyond its strict validity range, but as argued before and in previous papers, the semi-quantitative features are thought of to be reliable, provided $U$ is not too large. An example of this spectral density is shown in the case of $\mathrm{Ni}$ for $U=2 \mathrm{eV}$ in figure 2, but similar trends are observed for $\mathrm{Co}$ and $\mathrm{Fe}$. A bandlike state can always be identified and there is an additional structure at the bottom of the band, whose position in this case agrees with that of the observed satellite. From such a spectral density, it is then natural to redefine an effective self energy $\sum_{\sigma}^{\text {eff }}\left(E, U, N_{\mathrm{e}}\right)$, the real and imaginary parts of which are given by the measured shifts and widths (FWHM) of the d band-like states. Within these notations : $\Sigma_{\sigma}^{\text {eff }}\left(E, U, N_{\mathrm{e}}\right) / U^{2} \rightarrow \Sigma_{\sigma}\left(E, N_{\mathrm{e}}\right)$ as $U \rightarrow 0$. In order to get a general trend (independent of $U$ and $N_{\mathrm{e}}$ ) for the energy dependence of $\Sigma_{\sigma}^{\text {eff }}$, it is convenient to separate the contributions of the different factors $\left(U, N_{\mathrm{e}}, E\right)$. This can be done empirically as follows :

$$
\begin{aligned}
\Sigma_{\sigma}^{\text {eff }}(E, U, & \left.N_{\mathrm{e}}\right)=U^{2}\left(\Sigma_{\sigma}^{0}\left(N_{\mathrm{e}}\right)+\right. \\
& \left.+\left(N_{\mathrm{e}} / 10\right)\left(1-N_{\mathrm{e}} / 10\right) g_{\sigma}\left(N_{\mathrm{e}}\right) S_{\sigma}(E, U)\right)
\end{aligned}
$$
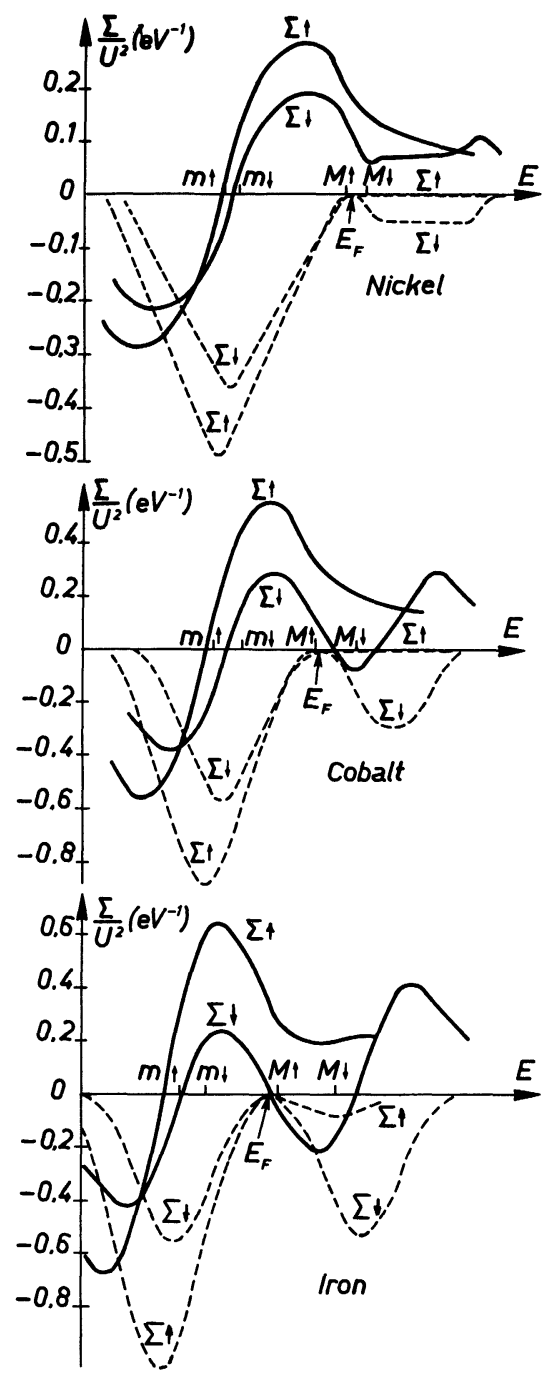

Fig. 1. - Energy dependence of the self energy as a function of the $\mathrm{d}$ band filling : $N_{\mathrm{e}}=9.4(\mathrm{Ni}), N_{\mathrm{e}}=8.4(\mathrm{Co})$ and $N_{\mathrm{e}}=7.3(\mathrm{Fe}) . \longrightarrow \operatorname{Re} \Sigma_{\sigma}\left(E, N_{\mathrm{e}}\right) ; \cdots \operatorname{Im} \Sigma_{\sigma}\left(E, N_{\mathrm{e}}\right.$ ) (units are $\left.\mathrm{eV}^{-1}\right)$.

by analogy with the quantity that gives the order of magnitude of $\Sigma\left(E, U, N_{\mathrm{e}}\right)$. Such a separation has been successfully tested for reasonable values of $U$ $(0<U \leqslant 2.5 \mathrm{eV})$ and for the fillings of interest here. Furthermore, these different tests have shown that, except at the very bottom of the band for very small values of $U$, it could be assumed that : $S_{\sigma}(E, U) \simeq S_{\sigma}(E)$. This is shown in figure 3 , where $S_{\sigma}(E)$ is given by the solid lines, whereas $S_{\sigma}(E, U)$ would be given by a similar line varying between the dashed lines (shaded areas) for the values of $U$ mentioned above, so that the shaded areas in figure 3 represent the uncertainty due to the assumption : $S_{\sigma}(E, U) \simeq S_{\sigma}(E)$. Therefore, we have now a reduced self energy $S_{\sigma}(E)$ which depends solely on energy and whose overall behaviour gives us qualitative general results, independently of the values of $U$ and of the filling of the $\mathrm{d}$ band. Quantitative results are obtained by inserting $S_{\sigma}(E)$ in formula (6), using the values of the table I for $g_{\sigma}$ and $\Sigma_{\sigma}^{0}$. 


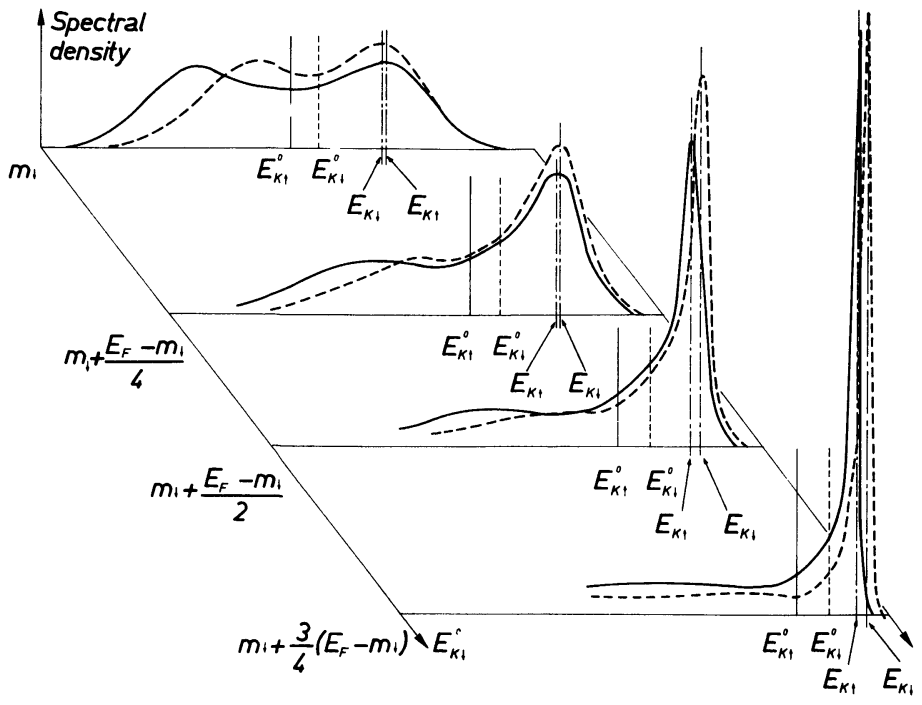

Fig. 2. - Spectral density of $\mathrm{Ni}: E_{k \sigma}^{0}$ is the initial Bloch state $(U=0)$ and $E_{k \sigma}$ the band-like state when correlations are taken into account $(U=2 \mathrm{eV}):-\sigma=\uparrow ;-\cdots---\sigma=\downarrow$.

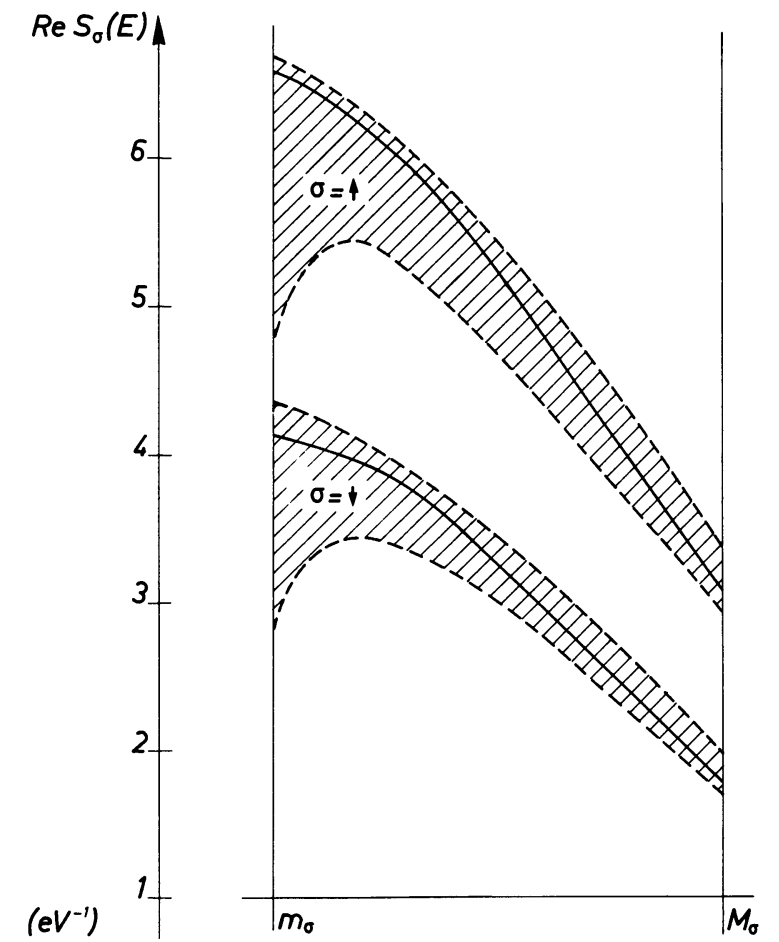

Fig. 3. - Energy dependence of the "reduced" shift $\operatorname{Re} S_{\sigma}(E)$. The effective shifts are obtained by using formula (6) with values from table I.

The behaviour of the real part of $S_{\sigma}(E)$, shown in figure 3 , leads to a narrowing of the bands $\left(\operatorname{Re} S_{\sigma}(E)\right.$ decreases from $m_{\sigma}$ to $M_{\sigma}$ ) and also to a reduction of the exchange splitting $\left(S_{\uparrow}\left(E_{k \uparrow}\right)>S_{\downarrow}\left(E_{k \downarrow}\right)\right)$ which is essentially due the factors 4 and 5 in formula (5). The imaginary part of $S_{\sigma}(E)$ has a similar variation. This implies that the spectral peaks are much larger
Table I. - Parameters used in formula (6) for $\mathrm{Ni}, \mathrm{Co}$ and $\mathrm{Fe}$.

\begin{tabular}{|c|c|c|r|r|r|r|}
\cline { 2 - 7 } \multicolumn{1}{c|}{} & \multicolumn{2}{c|}{$\mathrm{Ni}\left(N_{\mathrm{e}}=9.4\right)$} & \multicolumn{2}{c|}{$\mathrm{Co}\left(N_{\mathrm{e}}=8.4\right)$} & \multicolumn{2}{c|}{$\mathrm{Fe}\left(N_{\mathrm{e}}=7.3\right)$} \\
\cline { 2 - 7 } \multicolumn{1}{c|}{} & $\sigma=\uparrow$ & $\sigma=\downarrow$ & $\sigma=\uparrow$ & $\sigma=\downarrow$ & $\sigma=\uparrow$ & $\sigma=\downarrow$ \\
\hline$g_{\sigma}\left(N_{\mathrm{e}}\right)$ & 1 & 1 & 0.9 & 1.4 & 0.7 & 1.1 \\
\hline $\begin{array}{c}\Sigma_{\sigma}^{0}\left(N_{\mathrm{e}}\right) \\
\left(\mathrm{eV}^{-1}\right)\end{array}$ & 0 & 0 & -0.07 & -0.42 & -0.12 & -0.62 \\
\hline
\end{tabular}

for lower than upper states (see for example the case of $\mathrm{Ni}$ discussed in section $\S 4.1$ ).

We now introduce correlations in one electron band structures, by adding to the $\mathrm{d}$ component of each initial Bloch state energy $E_{k \sigma}^{0}$, the real part of the effective self energy $\Sigma_{\sigma}^{\text {eff }}\left(E_{k \sigma}^{0}\right)$, i.e. we assume that correlation effects are negligible for sp states. To this end it is convenient to reproduce these unperturbed bands by using an interpolation scheme (see for example Ref. [29]). Such a technique is well suited to the tight binding formalism since it yields directly the weights of the $\mathrm{d}$ and sp parts of the Bloch states. Then these states are corrected by adding to $E_{k \sigma}^{0}$ the real part of $\Sigma_{\sigma}^{\text {eff }}\left(E_{k \sigma}^{0}\right)$ multiplied by the weight of the d part. In practice the latter has been assumed to be proportional to the splitting of the band of interest, which is quite consistent with the spirit of interpolation schemes [29]. A more exact procedure would be to insert the self energy into the $d$ part of this scheme and to recalculate the spectral density of states, but this would induce minor differences. Another possible procedure would be that used by Davis and Feldkamp [14] : they have added the real part of the self energy to the $d$ part of the hamiltonian and diagonalized this new full hamiltonian. Then it remains to redetermine $E_{\mathrm{F}}$, since our calculation is not self-consistent : this is done by conserving the number of $\mathrm{d}$ electrons $N_{\mathrm{e}}$. As a result, the corrected band structures (described in the following paragraph) reproduce more accurately than the initial ones the experimental results, at least for $\mathrm{Ni}$ and $\mathrm{Co}$.

One can also inquire whether these corrections could account for the discrepancies found in one electron band calculations, concerning the electronic specific heat $C_{v}$. In fact, the measured specific heat includes not only a one electron contribution, but also terms related to many body effects, and $C_{\mathrm{v}}$ is generally written as :

$$
C_{\mathrm{v}}=\gamma T \text { with }: \quad \gamma=\gamma_{0}(1+\lambda)
$$

and :

$$
\gamma_{0}=\frac{\pi}{3} k_{\mathrm{B}}^{2} N_{\mathrm{bs}}\left(E_{\mathrm{F}}\right)
$$

where $N_{\mathrm{bs}}\left(E_{\mathrm{F}}\right)$ is the one electron density of states at the Fermi level and $\lambda$ the so-called enhancement 
factor which contains two main additive contributions : electron-phonon $\lambda_{\mathrm{e}-\mathrm{p}}$ and electron-electron $\lambda_{\mathrm{e}-\mathrm{e}}$.

Systematic calculations of $\lambda_{\mathrm{e}-\mathrm{p}}$ have been performed by Papaconstantopoulos et al. [30] for 32 metals with $Z \leqslant 49$. The resulting values are not sufficient to account for the total $\lambda$ [31-32] except in the case of noble metals $\mathrm{Ag}, \mathrm{Cu}$. As can be seen in table II, the discrepancies are rather large $\left(\lambda-\lambda_{\mathrm{e}-\mathrm{p}} \sim 0.5\right)$. As a rule [33-34], $\lambda$ is related to the full self energy through :

$$
\lambda=-\left.\operatorname{Re}\left(\frac{\mathrm{d} \Sigma}{\mathrm{d} E}\right)\right|_{E_{\mathbf{F}}} .
$$

Table II. - Comparison between experimental [31, 32] and [22] and theoretical [30] values of the enhancement factor of the electronic specific heat.

\begin{tabular}{|c|c|c|c|c|c|}
\cline { 2 - 6 } \multicolumn{1}{c|}{} & $\mathrm{Fe}$ & $\mathrm{Co}$ & $\mathrm{Ni}$ & $\mathrm{Cu}$ & $\mathrm{Ag}$ \\
\hline$\lambda[31,32]$ experiments & 1.03 & 0.78 & 0.77 & 0.022 & 0.011 \\
\hline$\lambda_{\mathrm{e}-\mathrm{p}}[30]$ & 0.52 & 0.38 & 0.17 & 0.058 & 0.051 \\
\hline$\lambda-\lambda_{\mathrm{e}-\mathrm{p}}$ & 0.5 & 0.4 & 0.6 & -0.03 & -0.04 \\
\hline$\lambda_{\mathrm{e}-\mathrm{e}} / U^{2}$ & 0.20 & 0.13 & 0.11 & 0 & 0 \\
\cline { 2 - 6 } \\
$\begin{array}{c}\text { from figure 1 and } \\
\text { formula (8)) }\left(\mathrm{eV}^{-2}\right)\end{array}$
\end{tabular}

Now, we show that the main contribution to $\lambda_{\mathrm{e}-\mathrm{e}}$ comes from correlation effects and that the previous discrepancies can be explained by invoking them. Actually, using the results described in figure 1 , we do find an enhancement (i.e. $\left.\frac{\mathrm{d} \Sigma}{\mathrm{d} E}\right|_{E_{\mathrm{F}}}<0$ as it should be from Landau theory) with the good order of magnitude for $U$ about $1-2 \mathrm{eV}$; this will be discussed in more details in the next section. Furthermore, it is satisfactory to verify that the calculated $\lambda_{\mathrm{e}-\mathrm{p}}$ account practically for the whole enhancement $\lambda$ of $\mathrm{Cu}$ and $\mathrm{Ag}$, since within our scheme, $\lambda_{\mathrm{e}-\mathrm{e}}$ vanishes for a full $\mathrm{d}$ band (see formula (5)).

To sum up, introducing correlations in one electron band calculations, in the case of transition metals with unfilled $\mathrm{d}$ bands, always leads to a reduction of the $\mathrm{d}$ bandwidth, a diminution of the exchange splitting, a broadening of the spectral lines (principally for lower $d$ states), an enhancement of the specific heat and to the appearance of a satellite, the intensity of these effects being roughly proportional to $\left(N_{\mathrm{e}} / 10\right)\left(1-N_{\mathrm{e}} / 10\right) U^{2} / W^{2}$ as previously explained.

4. Corrected band structures of $\mathrm{Ni}$, Co and $\mathrm{Fe}$. 4.1 NiCKel. - We first apply the formalism developed in previous sections to the case of $\mathrm{Ni}$ which has initiated the whole series of papers on correlations in transition metals. We have already discussed [18], within our perturbation method, the intensity and the position of the satellite which appears $6 \mathrm{eV}$ below the Fermi level : this led to $U \simeq 2 \mathrm{eV}$. We now examine the band structure of ferromagnetic nickel.

The self energies $\Sigma_{\uparrow}$ and $\Sigma_{\downarrow}$ shown in figure 1 have been calculated using formula (5) with the following parameters [20-22] :

$N_{\mathrm{e}}=9.4 ; \mu=0.6 ; \quad W_{\downarrow}=4.2 \mathrm{eV} ; \quad W_{\uparrow}=4.0 \mathrm{eV}$.

Very recently, Kleinman and Mednick have calculated the full band and wave-dependent second order self energies in ferromagnetic Ni [16]. Their results compare very closely with ours, which is a nice justification of our approximations (i.e. local approximation and rectangular density of states), the relative fluctuations of $\Sigma_{k}(E)$ as a function of $k$ being of the order of $10 \%$. On the other hand, Liebsch has also obtained similar results by using a different approach. In the low density limit [11] the slope of its self energy differs from ours, being peaked at much larger binding energies. In a subsequent paper [12], he argues that one should go beyond this limit and he sets up an improved theory that yields better agreement with experiments concerning the satellite and the narrowing of the $d$ band. Its new self energy is then skewed towards smaller binding energies and actually does not appreciably differ from that shown in figure 1.

We now apply the procedure described in $\S 3$, i.e. we calculate the spectral densities $n_{k \sigma}\left(E_{k \sigma}\right)$ for different values of $U$ (see for example the case $U=2 \mathrm{eV}$ in figure 2) and deduce the effective self energies $\Sigma_{\sigma}^{\text {eff }}\left(E, U, N_{\mathrm{e}}\right)$ (Fig. 3, table I). It can be seen in figure 2 that the satellite appears for each value of $E_{k \sigma}$, nearly at the same position (there is no dispersion), with an intensity which increases when going deeper in the band. The shifts $\operatorname{Re} \Sigma_{\sigma}^{\text {eff }}$ are roughly linear functions of the initial energies in the upper part of the band :

$$
\operatorname{Re} \Sigma_{\sigma}^{\mathrm{eff}}\left(E_{k \sigma}^{0}\right)=\alpha_{\sigma}\left(E_{\mathrm{F}}-E_{k \sigma}^{0}\right) .
$$

Using $U=2 \mathrm{eV}$ and averaging other both spin directions we find $\alpha=\bar{\alpha}_{\sigma} \sim 30 \%$. Similar values have been obtained by Davis and Feldkamp [14] and Liebsch [12]. This agrees with experimental data, as compiled by Eberhardt and Plummer [4] (see their figure 5). Notice that their data indicate a saturation effect at the bottom of the $\mathrm{d}$ band, which actually may also be observed in our figure 3 .

Let us now discuss the broadening of the band states related to $\operatorname{Im} \Sigma_{\sigma}^{\text {eff }}$. It obviously vanishes at $E_{\mathrm{F}}$ and increases up to about $1.5 \mathrm{eV}$ at the bottom of the band (for $U=2 \mathrm{eV}$ ), which is quite consistent with the experimental estimates $[4,35]$ as can be seen in figure 4 . The theoretical curve is slightly below the experimental values, but it should be kept in mind that the latter also include a contribution from final states.

We can now study the modifications induced by correlations on the one electron band calculations of 


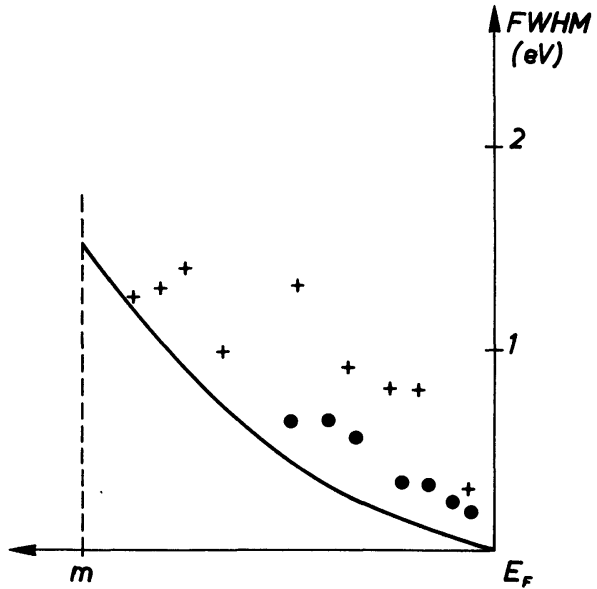

Fig. 4. - Comparison between the theoretical $\left(\operatorname{Im} \Sigma_{\sigma}^{\text {eff }}\right.$ $\left.\left(E, U=2 \mathrm{eV}, N_{\mathrm{e}}=9.4\right)\right)$ and experimental $[4,35]$ broadenings of the band states :- $\operatorname{Im} \Sigma^{\text {eff }} ;++++$ experiments [4];

Moruzzi et al. [22] by inserting $\operatorname{Re} \Sigma_{\sigma}^{\text {eff }}(E, U=2 \mathrm{eV}$, $N_{\mathrm{e}}=9.4$ ) in the interpolation scheme described in the previous section : let us point out that this simplified treatment gives only informations on the band structure and does not intend to reproduce complete angle-resolved photoemission spectra, which would require to enter the self energy (real and imaginary part) in a full photoemission calculation. This has been successively achieved by Desjonquères and Pendry [36] in the case of paramagnetic Ni. The modified band structure is shown in figure 5 for the same value of $U(U=2 \mathrm{eV})$. One sees that the agreement between theoretical and experimental bands is strongly improved when correlations are taken into account. This has already been shown by Davis and Feldkamp [14], but the agreement here is somewhat more satisfactory. In particular, we have a more realistic reduction of the splitting at $\mathrm{L}_{3}(0.3 \mathrm{eV}$ instead of $0.6 \mathrm{eV}$ for $U=0$ ) and the respective positions of points $\mathrm{L}_{2}^{\prime}$ and $\mathrm{L}_{3 \uparrow}$ are now consistent with experiments. This last point is essentially due to the fact that $L_{3}$ is a pure d state, whereas $L_{2}^{\prime}$ corresponds to a state made up of orthogonalized plane waves, i.e. to a pure non-d state. Thus, this state is not modified by correlations and the final effect is a negative shift relatively to $d$ states. Such a good agreement may be considered as somewhat fortuitous, since these points are very sensitive to variations of one electron potentials. However, almost all band calculations are in strong disagreement with the experimental data at these points, and the correction

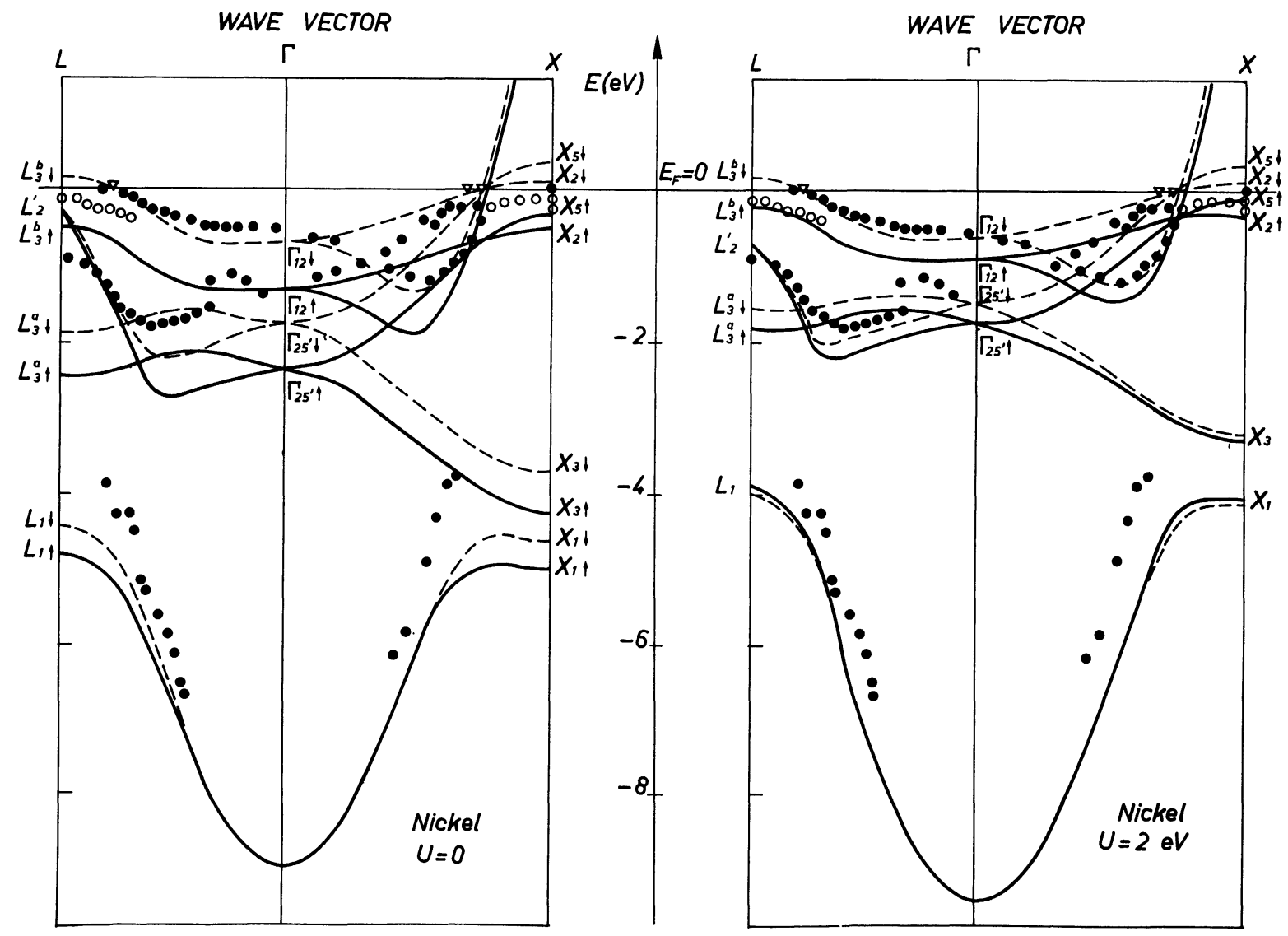

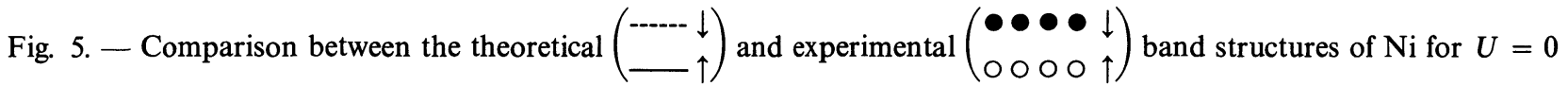
and $U=2 \mathrm{eV}$. One electron calculations $(U=0)$ are from reference [22] and experimental points from reference [8]. Triangles $\triangle$ denote de Haas van Alphen data. 
we found here is at least in the right direction. Note that recently relativistic effects have also been invoked to explain why $L_{2}^{\prime}$ is indeed below $L_{3}$ [20]. Davis and Feldkamp [14] mentioned a problem concerning $\mathrm{X}_{2}$ which is not discussed here since recent experiments by Eastman and Himpsel [8] seem to disagree with those of Eberhardt and Plummer [4] on this point.

Notice that we find the same value of the exchange splitting $(\sim 0.3 \mathrm{eV})$ at the uppermost $\mathrm{L}$ and $\mathrm{X}$ points and at $\mathrm{X}_{2}$, to be compared respectively to $0.32 \mathrm{eV}$ and $0.20 \mathrm{eV}$ in experiments [6,8]; as a consequence $\mathrm{X}_{2 \downarrow}$ is found slightly above $E_{\mathrm{F}}$ in our calculation whereas experimental minority and majority bands are occupied at this point. Eastman and Himpsel [8] explain these small differences by invoking a symmetry dependence of the correlation effects [12] at points $X_{2}$ and $X_{5}$, which are states with a pure $e_{g}$ and $t_{2 g}$ character respectively. We do not think so; actually, calculating a symmetry dependent self energy $\Sigma_{\mathrm{t}_{2 \mathrm{~g}}}\left(\right.$ or $\left.\Sigma_{\mathrm{e}_{\mathrm{g}}}\right)$ requires a triple integration involving both bare densities of states $n_{\mathbf{t}_{2 \mathbf{g}}}(E)$ and $n_{\mathrm{e}_{\mathrm{g}}}(E)$. A precise formulation can be obtained by extending the calculation described in reference [17] for the total energy. The final result is that : $\Sigma_{\mathrm{t}_{2}} \simeq \Sigma_{\mathrm{e}_{\mathrm{g}}}$. On the contrary, and as far as the unperturbed state is concerned, it should be realized that any interpolation scheme based on a tight binding description of the $\mathrm{d}$ bands should yield in Ni splittings which are strongly symmetry dependent : within the Stoner model, the splittings are directly proportional to the number of holes (i.e. a single integration over $n_{\mathbf{t}_{2 \mathrm{~g}}}(E)$ or $n_{\mathrm{e}_{\mathrm{g}}}(E)$ is required) and the d holes in $\mathrm{Ni}$ are mostly $t_{2}$ so that we expect larger splittings for this symmetry (at least within this model) [29]. The tight binding model probably overestimates this effect, but it might be that the self-consistent calculations which use muffin tin potentials underestimate it. Anyway, we do not expect correlation effects to introduce more symmetry effects than included in the initial one electron approximation.

When correlations are taken into account, the splitting remains an increasing function of the energy as can be seen in figure 5. Our corrections are obviously not very realistic at the bottom of the band, because of the very large width of the peaks. Actually, except near the Fermi level, separate up and down spin peaks have not yet been resolved in experiments.

We have already mentioned the recent work of Kleinman and Mednick [16]. They have chosen for $U$ the value which gives the best fit to experiments for the splitting at the Fermi level. We think that it is more appropriate to use more global properties such as the narrowing of the bands. Taking $U=1.14 \mathrm{eV}$ as they did would lead to a rather weak narrowing $(\alpha \sim 10 \%)$. Furthermore, no satellite at all would be obtained.

Thus, we have shown that using a single value of the Coulomb integral $(U=2 \mathrm{eV})$, we can explain all the features observed in photoemission spectra : position and intensity of the satellite, narrowing of the $\mathrm{d}$ bandwidth $(\sim 30 \%)$, reduction of the splitting ( $\sim 50 \%$ ), width of the spectral lines, and even enhancement of the specific heat (inserting $U=2 \mathrm{eV}$ in table II yields a reasonable value : $\lambda_{\mathrm{e}-\mathrm{e}} \simeq 0.44$ ). We show elsewhere [37] that the same value of $U$ also accounts for the Auger spectra.

4.2 Cobalt. - As explained previously, there is no reason for correlations to strongly affect the $\mathrm{Ni}$ band structure and not that of $\mathrm{Co}$, even if the effects are expected to be weaker in this latter case. $U$ is indeed expected to decrease when going from $\mathrm{Ni}$ to $\mathrm{Co}$ and $\mathrm{Fe}$ (although this is compensated in part by an increasing number of $d$ holes). In fact, there are only few experimental data on $\mathrm{Co}$ and the more detailed are those by Himpsel and Eastman [7]; they also show a narrowing of $\sim 20 \%$ of the $\mathrm{d}$ bandwidth and a reduction of $35 \%$ of the exchange splitting at the Fermi level, when compared to theoretical calculations of Moruzzi et al. [22]. Since our method is not limited to almost filled $\mathrm{d}$ bands, we can apply the same procedure as in the case of $\mathrm{Ni}$. The only problem comes from the lack of self-consistent one electron band calculations for HCP Co, which is the structure of $\mathrm{Co}$ at room temperature. However, such a calculation has been performed for FCC Co by Moruzzi et al. [22], from which we have estimated our input parameters :

$$
\begin{gathered}
N_{\mathrm{e}}=8.44 ; \quad \mu=1.56 ; \quad W_{\downarrow}=4.95 \mathrm{eV} ; \\
W_{\uparrow}=4.45 \mathrm{eV} .
\end{gathered}
$$

Let us recall that the exact self energies $\Sigma_{\sigma}$ are not expected to depend significantly on the crystalline structure. Therefore, our self energies shown in figure 1 and calculated with a rectangular density of states fitted to the above FCC parameters can be used in the case of HCP Co as well. Then we may calculate spectral densities that are quite similar to those of $\mathrm{Ni}$; in particular, we obtain a satellite with a weaker intensity for $U \geqslant 1 \mathrm{eV}$.

The corresponding real parts of the effective self energies are given in figure 3 and table $I$ : they obviously lead to a narrowing of the $\mathrm{d}$ band and to a reduction of the exchange splitting. On the other hand, we find here also that the imaginary part of $\Sigma_{\sigma}^{\text {eff }}$ is a monotonous increasing function of the binding energy.

The necessity of having a true HCP self-consistent one electron band calculation appears when we want to modify these bands by introducing the shifts $\operatorname{Re} \Sigma_{\sigma}^{\text {eff }}$.

The solution adopted by Himpsel and Eastman in the interpretation of their experimental results [7] on HCP Co was to unfold the HCP bands along $\Gamma$-A, in order to obtain bands along $\Gamma-\mathrm{A}-\Gamma$ similar to those along $\Gamma$-L in the FCC structure (see Fig. 6). This is not the only way to unfold these bands and 
WAVE VECTOR

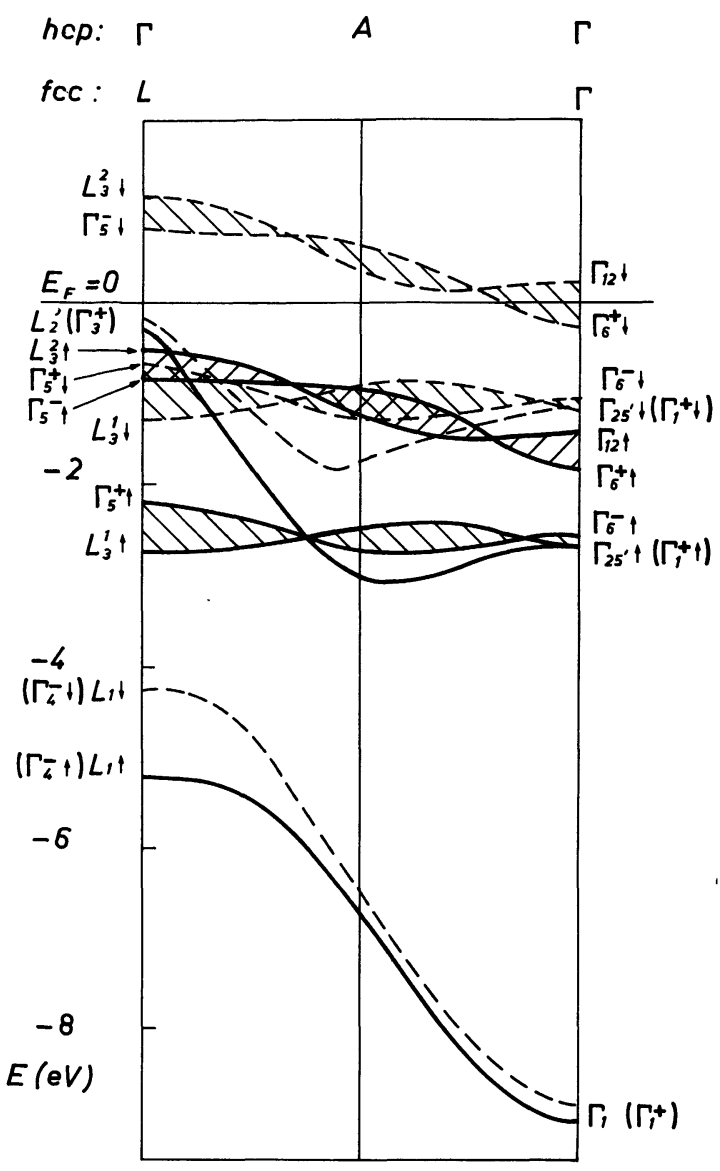

Fig. 6. - Unfolding of the HCP bands of Co along $\Gamma$-A in order to obtain FCC-like bands $\Gamma$-L. The shaded areas represent the errors made when assuming that $\mathrm{HCP}$ and FCC are identical. The symbols $\Gamma$ and $\mathrm{L}$ refer to FCC bands and $\Gamma^{+}$and $\Gamma^{-}$to HCP bands.

Himpsel and Eastman have chosen to conserve direct transitions; in other words, transitions from initial bands of one kind of symmetry to final states of the other kind (i.e. from initial states along $\Gamma$-A to final states along A- $\Gamma$ and vice versa) are forbidden. However, some problems remain since, although $\mathrm{HCP}$ and FCC are qualitatively equivalent in this direction, they are quantitatively different (compare in figure 6 the lines $\mathrm{L}_{3}^{2}-\Gamma_{12}$ and $\mathrm{L}_{3}^{1}-\Gamma_{25}$, to the lines $\Gamma_{5}^{-}-\Gamma_{6}^{+}$and $\left.\Gamma_{5}^{+}-\Gamma_{6}^{-}\right)$. Unfortunately, as already mentioned, there is no HCP self-consistent calculation and the method we have used is to transform the FCC bands of Moruzzi et al. [22] into HCP bands within an interpolation scheme with the same parameters in both crystalline structures. The differences between the two band structures are shown in figure 6 . Notice that the FCC band $\Gamma_{25},-\mathrm{L}_{2}^{\prime}$ that only involves $\mathrm{d}$ states of $3 z^{2}-r^{2}$ character $(\mathrm{O} z / /\langle 111\rangle)$ is unchanged under this transformation : it becomes $\Gamma_{1}^{+}-\Gamma_{3}^{+}$in HCP notations. The details of this transformation are given in the appendix. Another unfold- ing of the bands which could be more convenient for other purposes would be to preserve all the $\Gamma^{+}$ points at the centre of the zone. In this way bonding states are always located at $\Gamma$ [38].

We can now modify these quasi-self-consistent HCP one electron band structures by introducing the shifts $\operatorname{Re} \Sigma_{\sigma}^{\text {eff }}$ induced by correlations; this leads to the corrected band structure of figure 7 for

$$
U=1.5 \mathrm{eV} \text {. }
$$

The agreement between theory and experiment is less accurate here than in the case of $\mathrm{Ni}$, but is much more satisfactory, using $U=1.5 \mathrm{eV}$, than when correlation effects are omitted. In particular, we find a reasonable splitting at $E_{\mathrm{F}}(\sim 0.9 \mathrm{eV})$ and a narrowing of the $\mathrm{d}$ band of about $20 \%$. However, some problems remain, particularly concerning the position of $\Gamma_{3}^{+}$ which has not been sufficiently shifted down here (see the discussion concerning the equivalent point $\mathrm{L}_{2}^{\prime}$, in the case of Ni). Due to the energy dependence of the initial splitting $(U=0$; calculations of Moruzzi et al. [22]) we get a final weak dependence of this splitting : 0.8 to $0.9 \mathrm{eV}$ from the bottom to the top of the band, to be compared to that observed by Himpsel and Eastman [7], 1.2 to $0.9 \mathrm{eV}$. This decreasing trend is rather surprising and requires further studies. Notice that no important symmetry effects are expected here since the number of holes is roughly equivalent for the three types of point symmetry in HCP [39].

However, as a whole $U \simeq 1.5 \mathrm{eV}$ seems to be a reasonable value for Co. Actually it also accounts for several other properties :

i) widths of the spectral lines consistent with those observed by Himpsel and Eastman;

ii) enhancement of the specific heat : $\lambda_{\mathrm{e}-\mathrm{e}} \simeq 0.3$ instead of the estimated experimental value $\lambda_{\mathrm{e}_{-} \mathrm{e}} \simeq 0.4$ for FCC Co. The FCC structure has been chosen since no self-consistent calculation of the density of states for HCP is available. The experimental value for FCC Co is based on the extrapolation of experimental results on Ni-Co alloys [32];

iii) Auger spectra [37].

Finally, we want to point out that in our opinion, one should observe in Co a satellite similar to that of Ni. However, it should be broader (larger splitting) and weaker $(U / W$ smaller).

4.3 Iron. - We now consider the case of Fe. Unfortunately very few experimental data are available at the moment $[25,40]$ and a significant comparison between theory and experiment can hardly be made. It seems also that there are problems for unambiguously identifying direct transitions [41]. A first study seems to indicate that there is a rather fair agreement between the experimental points by Eastman et al. [25] and BCC one electron calculations of Callaway and Wang [42], and therefore that correlations do not play an important rôle here. This is 


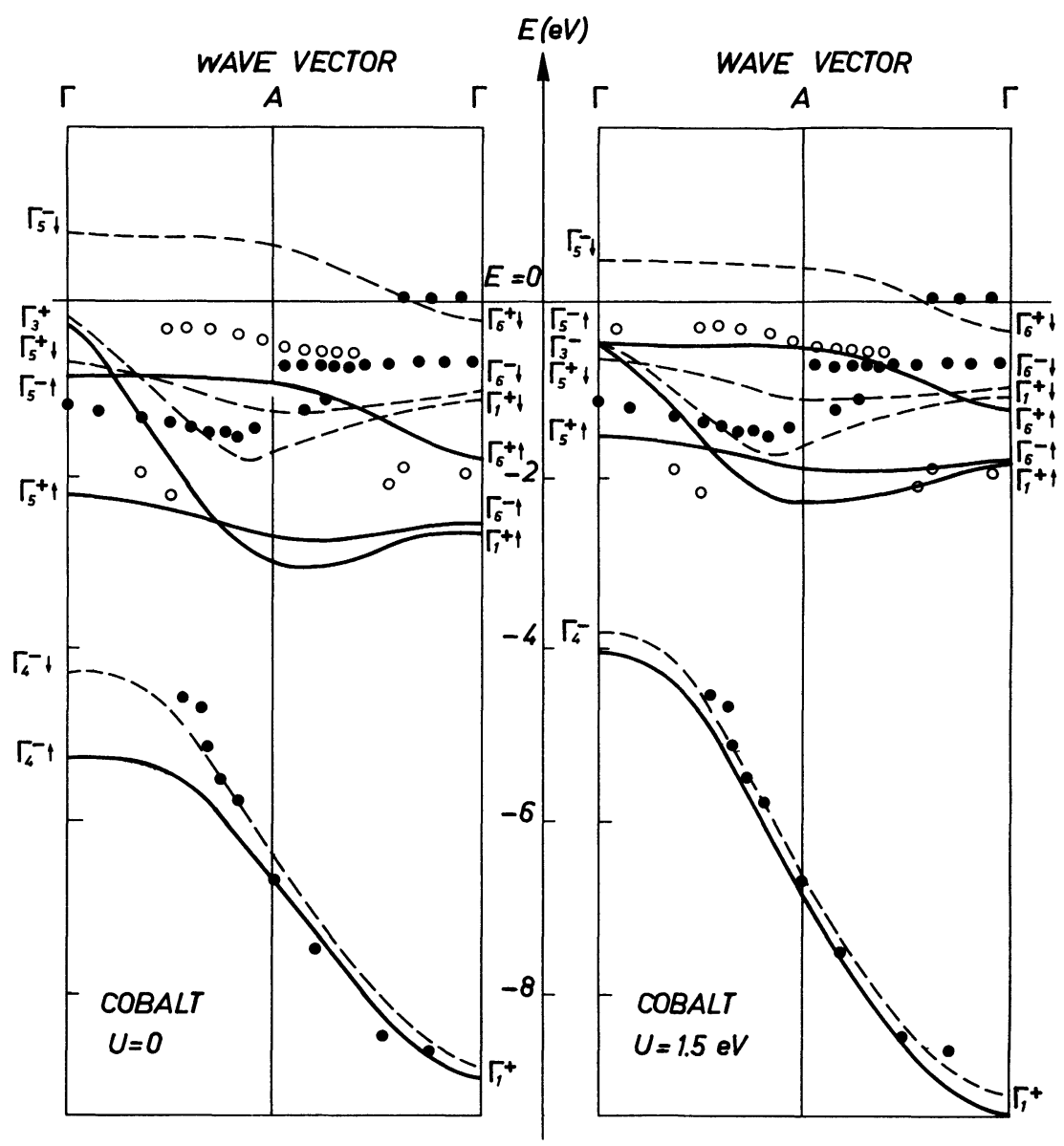

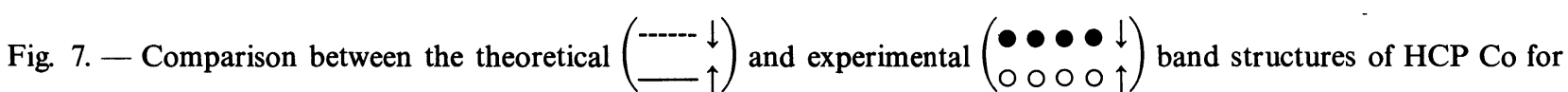
$U=0$ and $U=1.5 \mathrm{eV}$. Theoretical HCP bands $(U=0)$ are deduced from FCC calculations of reference [22] as explained in the main text and in figure 6. Experiments are from reference [26].

consistent with a low value of $U$ in this case. Nevertheless it is interesting to apply the previous formalism to the case of a weak ferromagnet such as iron $\left(N_{\mathrm{e}}=7.3 ; \mu=2.15 ; W_{\downarrow}=6.3 \mathrm{eV} ; W_{\uparrow}=4.8 \mathrm{eV}\right)$. Our results are shown in figures 1,3 (table I) and 8 for $U=1 \mathrm{eV}$. As expected, the same qualitative effects as in $\mathrm{Ni}$ and $\mathrm{Co}$ are obtained, but they are weaker and roughly of the order of magnitude of the experimental uncertainty. Thus, it is difficult to claim that the introduction of correlation effects improves on the agreement between theory and experiment, even if it seems to be the case when considering some particular features as the $\mathrm{d}$ bandwidth narrowing $(\sim 10 \%$ for $U=1 \mathrm{eV})$ [25].

Anyway, this calculation shows that a value of about $1 \mathrm{eV}$ for $U$ (which also accounts for Auger spectra of $\mathrm{Fe}$ ) do not lead to any spectacular effect. On the other hand, the enhancement of specific heat induced by correlations $\left(\lambda_{\mathrm{e}-\mathrm{e}}=0.2\right.$ instead of 0.5$)$ is underestimated when compared to the case of $\mathrm{Ni}$ and Co.

The necessity of using such a low value of $U$ to account for photoemission experiments is rather surprising when considering other physical properties [43]. For example, local moments seem to be very stable which indicates that intra-atomic correlations are important [44]; this could explain, according to some authors [45], the difficulty in obtaining unambiguous direct transitions. Recall however that we have neglected explicit exchange effects. Anyway, using larger values of $U$ would induce dramatic effects on single particle excitation spectra. Admitly perturbation theory breaks down in this case (more precisely, with $U \sim W$ the factor $N_{\mathrm{e}}\left(10-N_{\mathrm{e}}\right)(U / W)^{2}$ would be much larger than in $\mathrm{Ni}$ and $\mathrm{Co}$ ), but it is difficult to imagine that similar effects would not occur within a better theory.

5. Conclusion. - We have presented here a systematic study of the influence of correlations on band structure calculations for $\mathrm{Ni}, \mathrm{Co}$ and $\mathrm{Fe}$ within a perturbation treatment limited to second order in $U / W$, but the main conclusions discussed in the text are thought of to be more generally valid. Very good agreement with experiments is obtained in the case of $\mathrm{Ni}$ and to a lesser extend in the case of $\mathrm{Co}$; it is 


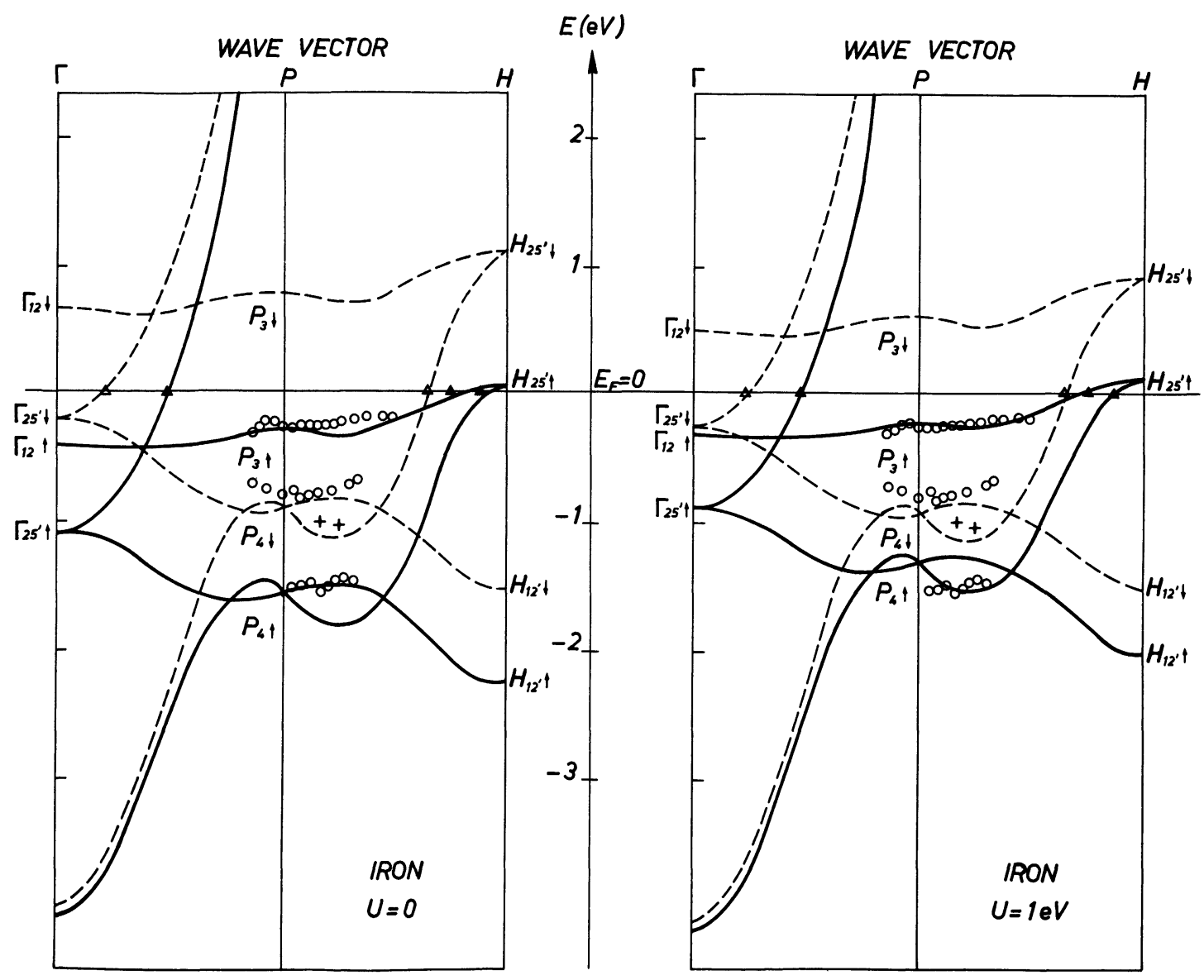

Fig. 8. - Comparison between the theoretical $\left(\begin{array}{c}----\downarrow \\ \downarrow\end{array}\right)$ and experimental $(\circ \circ \circ \bigcirc \bigcirc)$ band structures of $\mathrm{Fe}$ for $U=0$ and $U=1 \mathrm{eV}$. Theoretical one electron calculations $(U=0)$ are from reference [41] and experiments from reference [25].

more difficult to conclude in the case of Fe. As a whole, this is very satisfactory since we have used a very simple model, with only one adjustable parameter $U$ which accounts for several discrepancies found in photoemission spectra, and also in low temperature specific heats and Auger spectra.

Finally, let us comment on some remaining problems. The range of validity of our perturbation theory has already been discussed on several occasions [18] and the difficulties related to the determination of $U$ for $\mathrm{Fe}$ have been mentioned in $\S 4.3$. However, there is still the problem of a clear characterization of the unperturbed state. Actually the Hartree-Fock approximate solution of the Hubbard model and the band calculations we have used, based on the local density functional method, include average exchange and correlation contributions which are not directly comparable. In practice, different choices of this unperturbed state would induce differences in the relative positions of $\mathrm{d}$ and non-d states, and in the precise value of $U$.

These points are directly related to some problems mentioned by the referees. As a whole they argue that our values of $U$ are surprisingly small, which can be seen by calculating the corresponding exchange splitting within Hartree-Fock theory. This leads to $\Delta \simeq 0.24 \mathrm{eV}$ for $\mathrm{Ni}$, using $U=2 \mathrm{eV}$, i.e. to a splitting already smaller than the experimental one. Thus in some respect our corrections are not consistent with the ground state determined within the HartreeFock scheme. This is exactly the problem mentioned above. Now, from our discussion, we do think that some points have been clearly established : namely that any many body treatment of correlations should yield corrections to the ground state and excitation spectra, whose signs are unambiguous and which are qualitatively similar to those deduced from the comparison of band structure calculations with experiments. Furthermore there is a semi-quantitative agreement for these corrections by using $U$ values about 1-2 eV. It might be that a more detailed theory, going beyond perturbation theory, would lead to similar conclusions using larger values of $U$. This is what is suggested by a recent calculation by Igarashi [46] that includes electron-magnon interactions. This leads us back to the difficult (and old) problem of determining what kinds of correlations are included (or not) when using hamiltonians of the Hubbard type and $U$ values obviously smaller than the bare atomic ones [47]. 
Acknowledgments. - It is a pleasure to thank Dr. M. C. Desjonquères for stimulating discussions, Dr. D. Chandesris for communicating us unpublished results on nickel, and the referees for their useful comments (particularly for drawing our attention to the reference [46]).

Appendix : Unfolding of the HCP bands. - Using simple axis transformations, we can compare the wave vector $(k)$ dependence of the energy along $\Gamma$-L and $\Gamma$-A for FCC and HCP bands respectively, in

terms of the fixed following parameters : crystalline field $\left(E_{\mathrm{t}_{2 \mathrm{~g}}}\right.$ and $\left.E_{\mathrm{e}_{\mathrm{g}}}\right)$ and Slater $(\mathrm{dd} \sigma, \mathrm{dd} \pi$ and $\mathrm{dd} \delta)$ integrals. In practice, these parameters have been fitted so as to reproduce the results of Moruzzi et al. [22] for FCC Co at points $\Gamma$ and X. Actually, interpolation schemes provide simple expressions for the energy levels as a function of the $\mathrm{d}$ band parameters, at points $\Gamma_{12}, \Gamma_{25^{\prime}}, \mathrm{X}_{2}, \mathrm{X}_{3}$ and $\mathrm{X}_{5}$ [29]. Notice that the self-consistent calculations produce a non rigid splitting which necessits two sets of values corresponding to the two spin directions :

$$
\begin{array}{llll}
\sigma=\uparrow: E_{\mathrm{e}_{\mathrm{z}}}=7.656 \mathrm{eV} ; & E_{\mathrm{t}_{2 \mathrm{~g}}}=7.901 \mathrm{eV} ; & \mathrm{dd} \sigma=-0.552 \mathrm{eV} ; & \mathrm{dd} \pi=0.309 \mathrm{eV} \\
& & \mathrm{dd} \delta=-0.048 \mathrm{eV}
\end{array}
$$

Now the problem is that $k$ runs from 0 to $\pi / a$ for the FCC structure and from 0 to $\pi /(2 a)$ for the HCP one, $a$ being the distance between close-packed planes. Evidently, we have twelve bands, ten of which are pure d bands, in the latter case (two atoms per unit cell), but a comparison between the two structures can be made by unfolding the HCP bands about A, while preserving the vertical optical selection rules. It is easily realized that the FCC bands $\Gamma_{1}-\mathrm{L}_{1}$ and $\Gamma_{25},-\mathrm{L}_{2}^{\prime}$ are unchanged under this transformation; they become $\Gamma_{1}^{+}-\mathrm{A}_{1}-\Gamma_{4}^{-}$ and $\Gamma_{1}^{+}-\mathrm{A}_{1}-\Gamma_{3}^{+}$respectively (see Fig. A). As for the other bands (which are all doubly degenerate), they are obtained from the following formula :

$$
E^{i}(q)=\left(a^{i}+\varepsilon b^{i} \cos (q) \pm \sqrt{c^{i}+\varepsilon d^{i} \cos (q)+e^{i} \cos ^{2}(q)}\right) / 2
$$

where $i=1(2)$ corresponds to the FCC (HCP) structure, whereas the \pm sign corresponds to the band degeneracy and :

$$
\begin{aligned}
a^{1} & =a^{2}=E_{\mathrm{t}_{2 \mathrm{~g}}}+E_{\mathrm{e}_{\mathrm{g}}}+(9 \mathrm{dd} \sigma+24 \mathrm{dd} \pi+15 \mathrm{dd} \delta) / 4 \\
b^{1} & =(9 \mathrm{dd} \sigma+16 \mathrm{dd} \pi+23 \mathrm{dd} \delta) / 4 \\
b^{2} & =(21 \mathrm{dd} \sigma+8 \mathrm{dd} \pi-29 \mathrm{dd} \delta) / 12 \\
c^{1} & =\left(E_{\mathrm{t}_{\mathrm{g}}}-E_{\mathrm{e}_{\mathrm{g}}}+3(\mathrm{dd} \sigma-\mathrm{dd} \delta) / 4\right)^{2}+9(\mathrm{dd} \sigma-\mathrm{dd} \delta)^{2} / 2 \\
c^{2} & =\left(\left(E_{\mathrm{t}_{2 \mathrm{~g}}}-E_{\mathrm{e}_{\mathrm{g}}}\right) / 3+9(\mathrm{dd} \sigma-\mathrm{dd} \delta) / 4\right)^{2}+2(3 \mathrm{dd} \sigma-4 \mathrm{dd} \pi+\mathrm{dd} \delta)^{2} / 9 \\
d^{1} & =\left(E_{\mathrm{t}_{\mathrm{g}}}-E_{\mathrm{e}_{\mathrm{g}}}+3(\mathrm{dd} \sigma-\mathrm{dd} \delta) / 4\right)(3 \mathrm{dd} \sigma-8 \mathrm{dd} \pi+5 \mathrm{dd} \delta) / 2-9(\mathrm{dd} \sigma-\mathrm{dd} \delta)^{2} \\
d^{2} & =-\left(\left(E_{\mathrm{t}_{2 \mathrm{~g}}}-E_{\mathrm{e}_{\mathrm{g}}}\right) / 3+9(\mathrm{dd} \sigma-\mathrm{dd} \delta) / 4\right)(9 \mathrm{dd} \sigma+16 \mathrm{dd} \pi+23 \mathrm{dd} \delta) / 2 \\
e^{1} & =(3 \mathrm{dd} \sigma-8 \mathrm{dd} \pi+5 \mathrm{dd} \delta)^{2} / 16+9(\mathrm{dd} \sigma-\mathrm{dd} \delta)^{2} / 2 \\
e^{2} & =(9 \mathrm{dd} \sigma+16 \mathrm{dd} \pi+23 \mathrm{dd} \delta)^{2} / 16-2(3 \mathrm{dd} \sigma-4 \mathrm{dd} \pi+\mathrm{dd} \delta)^{2} / 9
\end{aligned}
$$
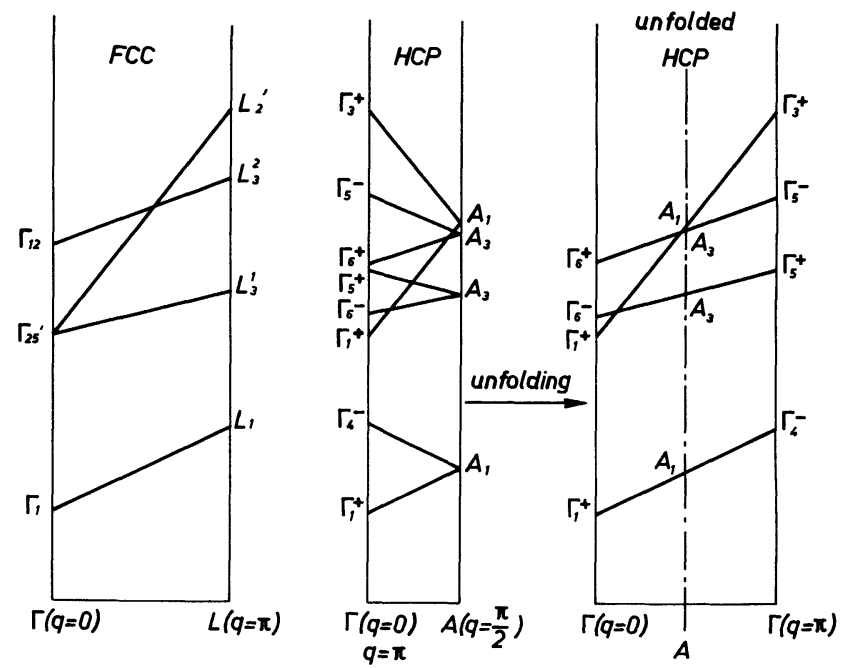

Fig. A. - Unfolding of schematic HCP bands. The exact relative positions of A points can be seen in figure 6 . 
In the case of the FCC structure $(i=1) \varepsilon=+1$ since there is only one atom per unit cell and $q=k a$ varies from $0(\Gamma)$ to $\pi(\mathrm{L})$. Then the sign + corresponds to the $\Gamma_{12}-\mathrm{L}_{3}^{2}$ line whereas the sign - corresponds to the $\Gamma_{25}, \mathrm{~L}_{3}^{1}$ one (see Fig. A).

On the other hand, in the case of the HCP structure, $q$ varies from $0(\Gamma)$ to $\pi / 2(\mathrm{~A})$ and $\varepsilon$ takes both values \pm 1 . Then, the present unfolding corresponds to set $\varepsilon=+1$ when going from $\Gamma(q=0)$ to $\mathrm{A}(q=\pi / 2)$ and $\varepsilon=-1$ from $\mathrm{A}(q=\pi / 2)$ to $\Gamma(q=\pi)$; simultaneously, in the latter case, $q$ has to be changed into $\pi-q$, which finally yields the following formula, valid for the FCC and the unfolded HCP bands :

$$
E^{i}(q)=\left(a^{i}+b^{i} \cos (q) \pm \sqrt{c^{i}+d^{i} \cos (q)+e^{i} \cos ^{2}(q)}\right) / 2 .
$$

In the HCP case $(i=2)$, the sign + corresponds to the $\Gamma_{6}^{+}-\mathrm{A}_{3}-\Gamma_{5}^{-}$line and the sign - to the $\Gamma_{6}^{-}-\mathrm{A}_{3}-\Gamma_{5}^{+}$one (see Fig. A).

From this discussion, it is clear that these bands in FCC and HCP structures have some parentage along $\Gamma$-L and $\Gamma-\mathrm{A}-\Gamma$, but due to the different values of the coefficients given in (A.2), they are not identical.

\section{References}

[1] Thiry, P., Chandesris, D., Lecante, J., Guillot, C., Pinchaux, R. and Petroff, Y., Phys. Rev. Lett. 43 (1979) 82.

[2] Stohr, J., Wehner, P. S., Williams, R. S., Apai, G. and Shirley, D. A., Phys. Rev. B 17 (1978) 587.

[3] Knapp, J. A., Himpsel, F. J. and Eastman, D. E., Phys. Rev. B 19 (1979) 4952.

[4] Eberhardt, W. and Plummer, E. W., Phys. Rev. B 21 (1980) 3245.

[5] Gerhardt, U., Maetz, C. J., Schutz, A. and Dietz, E., J. Magn. Magn. Mat. 15-18 (1980) 1141.

[6] Heimann, P. and Neddermeyer, H., J. Magn. Magn. Mat. $15-18$ (1980) 1143.

[7] Himpsel, F. J., KnAPP, J. A. and Eastman, D. E., Phys. Rev. B 19 (1979) 2919.

[8] Eastman, D. E. and Himpsel, F. J., Physics of Transition Metals 1980 (The Institute of Physics, Bristol and London) 1981, p. 115.

Himpsel, F. J., HeimanN, P. and Eastman, D. E., J. Appl. Phys. 52 (1981) 1658.

[9] Guillot, C., Ballu, Y., Paigne, J., Lecante, J., Jain, K. P., Thiry, P., Pinchaux, R., Petroff, Y. and Falicov, L. M., Phys. Rev. Lett. 39 (1977) 1632.

[10] Penn, D. R., Phys. Rev. Lett. 42 (1979) 921.

[11] Liebsch, A., Phys. Rev. Lett. 43 (1979) 1431.

[12] Liebsch, A., Phys. Rev. B 23 (1981) 5203.

[13] Davis, L. C. and Feldkamp, L. A., J. Appl. Phys. 50 (1979) 1944.

[14] Davis, L. C. and FeldKamp, L. A., Solid State Commun. 34 (1980) 141.

[15] Kleinman, L., Phys. Rev. B 19 (1979) 1295.

Kueinman, L., Phys. Rev. B 22 (1980) 6471.

[16] Kleinman, L. and Mednick, K., to be published.

[17] Treglia, G., Ducastelle, F. and SpanjaArd, D., J. Physique 41 (1980) 281.

[18] Treglia, G., Ducastelle, F. and Spanjaard, D., Phys. Rev. B 21 (1980) 3729.

[19] Treglia, G., Ducastelle, F. and Spanjaard, D., Phys. Rev. B 22 (1980) 6472.

[20] Anderson, J. R., Papaconstantopoulos, D. A., Boyer, L. L. and Schirber, J. E., Phys. Rev. B 20 (1979) 3172.

[21] Wang, C. S. and Callaway, J., Phys. Rev. B 15 (1977) 298.
[22] Moruzzi, V. L., Janak, J. F. and Williams, A. R., Calculated Electronic Properties of Metals (New York, Pergamon) 1978.

[23] Ishida, S., J. Phys. Soc. Japan 33 (1972) 369.

[24] Batallan, F., Rosenman, I. and Sommers, C. B., Phys. Rev. B 11 (1975) 545.

[25] Eastman, D. E., Himpsel, F. J. and Knapp, J. A., Phys. Rev. Lett. 44 (1980) 95 ; erratum 45 (1980) 498.

[26] Himpsel, F. J. and Eastman, D. E., Phys. Rev. B 21 (1980) 3207; erratum 22 (1980) 5014.

[27] Treglia, G., Ducastelle, F. and Spanjaard, D., Physics of Transition Metals 1980 (The Institute of Physics, Bristol and London) 1981, p. 45.

[28] Gunnarsson, O., Physica 91B (1977) 329.

[29] Ehrenreich, H. and Hodges, L., Methods in Computational Physics 8 (1968) 149.

[30] Papaconstantopoulos, D. A., Boyer, L. L., Klein, B. M., Williams, A. R., Moruzzi, V. L. and JanaK, J. F., Phys. Rev. B 15 (1977) 4221.

[31] Phillips, N. E., CRC Critical Reviews in Solid State Sciences 2 (1972) 467.

[32] Caudron, R., Meunier, J. J. and Costa, P., Solid State Commun. 14 (1974) 975.

[33] Luttinger, J. M., Phys. Rev. 119 (1960) 1153.

[34] SaCChetti, F., Solid State Commun. 30 (1979) 795.

[35] Chandesris, D., private communication.

[36] Desjonqueres, M. C. and Pendry, J. B., to be published.

[37] Treglia, G., Desjonqueres, M. C., Ducastelle, F. and Spanjaard, D., J. Phys. C 14 (1981) 4347.

[38] JoNES, H., The Theory of Brillouin Zones and Electronic States in Crystals (North-Holland Publishing Co : Amsterdam) 1960, p. 138.

[39] Hodges, L. and Ehrenreich, H., J. Appl. Phys. 39 (1968) 1280 ;

Moon, R. M., Phys. Rev. 136 (1964) A 195.

[40] Heimann, P. and Neddermeyer, H., Phys. Rev. B 18 (1978) 3537.

[41] Kevan, S. D., Wehner, P. S. and Shirley, D. A., Solid State Commun. 28 (1978) 517.

[42] Callaway, J. and Wang, C. S., Phys. Rev. B 16 (1977) 2095.

[43] Oles, A. M., Phys. Rev. B 23 (1981), 271 and private communication. 
[44] See for example the contributions by Korenman, Capellmann, Hasegawa and the Panel discussion on itinerant electron magnetism in the Proceedings of the International Conference on the Physics of Transition Metals (Leeds) published in : Physics of Transition Metals 1980 (The Institute of Physics, Bristol and London) 1981, p. 195, 205, 209, 669.
[45] Schulz, A., Courths, R., Schulz, H. and Hufner, S., J. Phys. F (Metal Phys.) 9 (1979) L-41.

[46] IGARASHI, J., Electron correlations in narrow bands, edited by T. Moriya (Springer) 1981, p. 115.

[47] KANAMORI, J., Electron correlations in narrow bands, edited by T. Moriya (Springer) 1981, p. 102. 